\title{
Measurement invariance of the Distress Tolerance Scale among university students with and without a history of non- suicidal self-injury
}

\author{
Ashley Slabbert ${ }^{1}$, Penelope Hasking ${ }^{1}$, Danyelle Greene ${ }^{1}$, Mark Boyes ${ }^{\text {Corresp. } 1}$ \\ ${ }^{1}$ School of Psychology, Curtin University, Perth, Western Australia, Australia \\ Corresponding Author: Mark Boyes \\ Email address: mark.boyes@curtin.edu.au
}

Non-suicidal self-injury (NSSI) is the intentional damage to one's body tissue in the absence of suicidal intent. NSSI primarily serves an emotion regulation function, with individuals engaging in self-injury to escape intense or unwanted emotion. Low distress tolerance has been identified as a mechanism that underlies self-injury, and is commonly assessed using the self-report Distress Tolerance Scale. There are mixed findings regarding the factor structure of the Distress Tolerance Scale, with some researchers utilising a higher-order distress tolerance score (derived from the scores on the four lowerorder subscales) and other researchers using the four subscales as unique predictors of psychological outcomes. Neither of these factor structures have been assessed among individuals with a history of self-injury. Of note, an inability to tolerate distress (thought to underlie NSSI) may limit an individual's capacity to accurately observe and report specific thoughts and emotions experienced in a state of heightened distress, which may impact the validity of scores on the Distress Tolerance Scale. Therefore, measurement invariance should be established before attributing NSSI-related differences on the scale to true differences in distress tolerance. We compared the Distress Tolerance Scale higher-order model with the lower-order four factor model among university students with and without a history of NSSI. Our results indicated that the lower-order four factor model was a significantly better fit to the data than the higher-order model. We then tested the measurement invariance of this lower-order factor model among individuals with and without a history of NSSI, and established configural and full metric invariance, followed by partial scalar and full residual error invariance. These results suggest the four subscales of the Distress Tolerance Scale can be used to confidently discern NSSI-related differences in distress tolerance. 
1 RUNNING HEAD: Measurement invariance of the Distress Tolerance Scale

2

3 Measurement Invariance of the Distress Tolerance Scale among University Students With and Without a History of Non-Suicidal Self-Injury

5

8 School of Psychology, Faculty of Health Sciences, Curtin University, Perth, Australia

11 *Corresponding author

12 Dr Mark Boyes

13 Associate Professor \& NHMRC Emerging Leadership Fellow

14 School of Psychology, Curtin University

15 GPO Box U1987, Perth, Western Australia, 6845

16 Phone: +61892667025

17 Fax: +61 892662464

18 Email: $\underline{\text { mark.boyes@,curtin.edu.au }}$

19 Web: www.markboyes.com

20 Twitter: @me_boyo \& @NSSI_RG

21

22

23 
Abstract

Non-suicidal self-injury (NSSI) is the intentional damage to one's body tissue in the absence of suicidal intent. NSSI primarily serves an emotion regulation function, with individuals engaging in self-injury to escape intense or unwanted emotion. Low distress tolerance has been identified as a mechanism that underlies self-injury, and is commonly assessed using the self-report Tolerance Scale, with some researchers utilising a higher-order distress tolerance score (derived from the scores on the four lower-order subscales) and other researchers using the four subscales as unique predictors of psychological outcomes. Neither of these factor structures have been assessed among individuals with a history of self-injury. Of note, an inability to tolerate distress (thought to underlie NSSI) may limit an individual's capacity to accurately observe and report specific thoughts and emotions experienced in a state of heightened distress, which may impact the validity of scores on the Distress Tolerance Scale. Therefore, measurement invariance should be established before attributing NSSI-related differences on the scale to true differences in distress tolerance. We compared the Distress Tolerance Scale higher-order model with the lowerorder four factor model among university students with and without a history of NSSI. Our results indicated that the lower-order four factor model was a significantly better fit to the data than the higher-order model. We then tested the measurement invariance of this lower-order factor model among individuals with and without a history of NSSI, and established configural and full metric invariance, followed by partial scalar and full residual error invariance. These results suggest the four subscales of the Distress Tolerance Scale can be used to confidently discern NSSI-related differences in distress tolerance. 
64

65

66

67

Peer] reviewing PDF | (2020:10:53519:1:0:NEW 5 Jan 2021) 
68

69

70

71

72

73

74

75

76

77

78

79

80

81

82

83

84

85

86

87

88

89

90

91

92

93

94

95

96

97

98

99

100

101

102

103

104

105

106

107

108

Non-suicidal self-injury (NSSI) is the intentional damage to one's body tissue in the absence of suicidal intent, for reasons not socially or culturally sanctioned (International Society for the Study of Self-Injury, 2018). International prevalence rates indicate approximately $13.4 \%$ of young adults report a history of self-injury, with elevated rates (20\%) reported by university students (Swannell, Martin, Page, Hasking, \& St John, 2014). NSSI is a behaviour receiving increasing attention from both researchers and clinicians, given its associations with negative psychological outcomes and heightened risk of suicide over time (Whitlock et al., 2013). Whilst there are various reasons for engaging in NSSI, individuals primarily report engaging in selfinjury for emotion regulation purposes (Taylor et al., 2018). Several key theoretical models of NSSI, including the Emotional Cascade Model (Selby, Anestis, \& Joiner, 2008), the Experiential Avoidance Model (Chapman, Gratz, \& Brown, 2006) and the Cognitive-Emotional Model (Hasking, Whitlock, Voon, \& Rose, 2017), specify a central role for emotion regulation in the onset and maintenance of self-injury. According to these models and previous empirical research, heightened negative affect (Armey, Crowther, \& Miller, 2011; Boyes, Wilmot, \& Hasking, 2019; Najmi, Wegner, \& Nock, 2007; Slabbert, Hasking, Notebaert, \& Boyes, 2020), low positive affect (Bresin, 2014; Slabbert et al., 2020; Victor \& Klonsky, 2014), greater repetitive negative thinking (Gong et al., 2019; Slabbert, Hasking, \& Boyes, 2018b), as well as greater difficulties in emotion regulation (Gratz, Breetz, \& Tull, 2010; Jenkins \& Schmitz, 2012) are all associated with increased likelihood of engaging in NSSI. Also common to these models is one's ability to tolerate distress arising from emotional experiences.

Distress tolerance refers to both an individual's perceived and actual ability to tolerate aversive physical and emotional states (Leyro, Zvolensky, \& Bernstein, 2010). Theoretically, individuals who experience greater difficulties tolerating intense emotion are less willing (or able) to withstand distress and more likely to self-injure as a means of escaping the aversive emotional state (Chapman et al., 2006). Researchers have established direct links between low distress tolerance and NSSI; individuals with lower levels of distress tolerance are more likely to report a history of self-injury (Anestis, Knorr, Tull, Lavender, \& Gratz, 2013; Lin, You, Wu, \& Jiang, 2018; Slabbert, Hasking, \& Boyes, 2018a) as well as more frequent NSSI (Anestis et al., 2013). Distress tolerance is typically assessed with self-report measures, most commonly the Distress Tolerance Scale (Simons \& Gaher, 2005).

The Distress Tolerance Scale (Simons \& Gaher, 2005) is a multidimensional scale designed to capture four core facets of distress tolerance; an individual's perceived ability to tolerate emotional distress (tolerance), subjective appraisal of distress regarding whether the distress is seen as acceptable or shameful (appraisal), the level of attention absorbed by distressing emotions (absorption), and efforts taken to alleviate the distress (regulation). A higher-order global distress tolerance score is derived by averaging the scores on the four subscales. The internal consistency of the higher-order scale and lower-order scales is generally good, with convergent and divergent validity previously established (Leyro, Bernstein, Vujanovic, McLeish, \& Zvolensky, 2010; Simons \& Gaher, 2005).

There is evidence to support the higher-order factor structure (Leyro, Bernstein, et al., 2010; Sandín, Simons, Valiente, Simons, \& Chorot, 2017; Werner-Seidler, Banks, Dunn, \& Moulds, 
109 2013), and many researchers opt to only utilise the total distress tolerance score in their research 110 (Anestis et al., 2013; Hovrud, Simons, Simons, \& Korkow, 2019; Peterson, Davis-Becker, \&

111 Fischer, 2014). However, there is growing acknowledgment that the tendency to only focus on

112 global distress tolerance has resulted in researchers losing potentially important information

113 captured in the individual subscales that may better explain relationships between distress

114 tolerance and psychopathology, or behaviours such as NSSI (Leyro, Bernstein, et al., 2010). In

115 studies where researchers have elected to investigate the four subscale scores, findings indicate

116 that some subscales may be more salient than others in predicting psychopathology such as

117 anxiety and depression, as well as dysregulated behaviours including self-injury (Horgan \&

118 Martin, 2016; Lin et al., 2018).

119 The two different factor structures have only been directly compared in one study. Among a

120 sample of Chinese adolescents, You and Leung (2012) found both the higher-order factor model

121 and lower-order four factor model demonstrated better fit than 1-factor and 2-factor structures

122 with which they were compared, with the higher-order model demonstrating best fit. Despite

123 You and Leung's (2012) findings, there is some evidence to suggest lower-order factor models

124 may demonstrate better model fit than higher-order models (Meganck, Vanheule, \& Desmet,

125 2008). Therefore, it is necessary to evaluate the fit of each of these models among individuals

126 with a history of NSSI to further our theoretical understanding of the nature of the relationship

127 between distress tolerance and self-injury, and consequently inform researchers about the

128 optimal way to utilise this scale with samples of individuals with a history of NSSI.

129 Another growing concern regarding the measurement of constructs such as distress tolerance, is

130 the accuracy of the heavily relied upon self-report scales such as the Distress Tolerance Scale to

131 detect true group differences. Researchers have become increasingly aware that statistically

132 observed differences on these scales are only meaningful when these instruments demonstrate

133 invariance across groups (Sass, 2016). Measurement noninvariance may have several

134 problematic implications. For example, using non-invariant scales to assess the severity of

135 psychological disorders such as depression across groups (i.e. women and men) may result in

136 one group (i.e. men) scoring lower than the other, simply because they interpret the items

137 differently, as opposed to actually experiencing less severe depression (Putnick \& Bornstein,

138 2016). Consequently, our understanding based on these findings is that females experience more

139 severe depression than men which may not be accurate, but directs future research towards

140 female-oriented studies and interventions (Putnick \& Bornstein, 2016). Relatedly, another

141 example of where measurement invariance is problematic is the use of pre-test and post-test

142 measurements to assess the effectiveness of an intervention or clinical trial. It is possible that the

143 intervention or trial itself may impact how participants interpret the constructs being assessed

144 (Putnick \& Bornstein, 2016). Consequently, this may result in inaccurate conclusions regarding

145 the effectiveness of an intervention (Putnick \& Bornstein, 2016). These example highlight the

146 importance of establishing measurement invariance in psychological science.

147 Recent research testing the measurement invariance of three emotion regulation questionnaires in

148 young adults with and without a history of NSSI showed that observed NSSI-related differences

149 on the Difficulties in Emotion Regulation Scale - Short Form (DERS-SF; Gratz \& Roemer, 
150 2004) and the Cognitive Emotion Regulation Questionnaire - Short (CERQ-S; Garnefski \&

151 Kraaij, 2007) were reliable and likely a true reflection of group differences in emotion regulation

152 (Kiekens, Hasking, \& Boyes, 2019). However, the widely used Emotion Regulation

153 Questionnaire (ERQ; Gross \& John, 2003) did not demonstrate measurement invariance, with

154 two of the items on the Cognitive Reappraisal subscale functioning differently for individuals

155 with a history of self-injury compared to individuals who had never self-injured. This is

156 concerning as previous research that has established NSSI-related differences in cognitive

157 reappraisal using this scale may be reflecting a measurement artefact rather than true group

158 differences. Similarly, Greene et al (2020) established that the Externally Oriented Thinking

159 subscale of the frequently used Toronto Alexithymia Scale (TAS-20; Bagby, Taylor, Parker, \&

160 Dickens, 2006) was not invariant across individuals with and without a history of NSSI,

161 precluding any conclusions regarding NSSI-related differences in externally oriented thinking.

162 These findings highlight the importance of investigating the measurement invariance of self-

163 report measures to reveal whether or not we can reliably draw conclusions about particular group

164 differences using these assessment tools.

165 Measurement invariance of the Distress Tolerance Scale between individuals with and without a

166 history of NSSI has not yet been assessed. It is plausible that the very difficulties in withstanding

167 distress underlying dysregulated behaviours such as NSSI, may limit an individual's capacity to

168 accurately observe and report specific thoughts and emotions experienced in a heightened

169 distressed state, which may impact the validity of results on self-report measures such as the

170 Distress Tolerance Scale. Observed differences in distress tolerance between people who do and

171 do not self-injure may be a function of a differential interpretation of scale items, rather than a

172 reflection of true group differences in distress tolerance. Given results derived from these

173 measures are used by researchers and clinicians to inform future prevention and intervention

174 programs, it is vital that we ensure these instruments are able to accurately produce reliable

175 results across individuals with and without a history of self-injury. Additionally, this may have

176 implications for the existing body of literature that has established NSSI-related differences in

177 self-report distress tolerance using this scale, and future researchers may need to be cautious

178 when using these findings to justify their aims or results.

179 This study had two primary aims. First, to test and compare the higher-order model of the

180 Distress Tolerance Scale to the lower-order four factor model to determine the best fitting model

181 among a sample of university students, as well as within subgroups of individuals with and

182 without a history of NSSI. Second, to test measurement invariance of the best fitting model

183 between individuals with and without a history of self-injury.

\section{Materials and Methods}

\section{Participants and procedure.}

186 Total Sample. Participants were 531 Australian University students ( $74.7 \%$ female) between the

187 ages of 17 and $25(\mathrm{M}=20.58, \mathrm{SD}=1.94)$ recruited through an undergraduate participant pool

188 and social media platforms. Of participants, 412 (77.6\%) were born in Australia, followed by

189 India (2.6\%), and Malaysia (2.3\%). The majority of participants were currently completing an 
190 undergraduate bachelor degree (96\%), followed by a Master degree (2.4\%). In total, $171(32.2 \%)$

191 of individuals reported a history of mental illness, most commonly anxiety and depression.

192 History of NSSI. Of the total sample, 215 individuals who reported a prior history of NSSI (Mage $193=20.87, \mathrm{SD}=2.0)$. Of these, $188(87.4 \%)$ were female, $173(80.5 \%)$ were born in Australia, and

$194202(94 \%)$ were studying an undergraduate bachelor degree. With regards to mental illness, 127

195 (59.1\%) participants reported a history of mental illness, most commonly anxiety and depression.

196 No history of NSSI. Of the total sample, 316 participants reported never engaging in NSSI (Mage

$197=20.38, \mathrm{SD}=1.88)$. Of these, 209 were female $(66.1 \%), 239(75.6 \%)$ were born in Australia,

198 and 308 (97.5\%) were studying an undergraduate bachelor degree. With regards to mental

199 illness, 44 (13.9\%) participants reported a history of mental illness, again most commonly

200 anxiety and depression.

201 After providing informed consent, participants completed a series of online questionnaires hosted

202 by Qualtrics. Data were collected as part of a larger study investigating the role of social,

203 cognitive, and emotional factors underlying health risk behaviours. Students received either

204 course credit or were entered into a prize draw to win an iPad or $\$ 50$ gift cards. This study

205 received ethical approval from the Curtin University Human Research Ethics Committee and

206 participants were provided with a list of counselling resources and information about self-injury

207 upon completion of the survey.

208 Measures.

209 Distress Tolerance. The 15-item Distress Tolerance Scale (Simons \& Gaher, 2005) was used to

210 assess individual differences in the ability to experience and withstand negative psychological

211 states. Items are rated on a 5-point Likert scale (1: strongly agree; 5: strongly disagree), with

212 higher scores reflecting higher levels of distress tolerance. The scale consists of four subscales:

213 tolerance (3 items, e.g. "I can't handle feeling distressed or upset"), appraisal (6 items, e.g. "My

214 feelings of distress or being upset are not acceptable"), absorption (3 items, e.g. "My feelings of

215 distress are so intense that they completely take over", and regulation (3 items, e.g. "I'll do

216 anything to avoid feeling distressed or upset"). Subscale scores are calculated by averaging

217 response to all items on each subscale. A higher-order distress tolerance score is calculated by

218 averaging the subscale mean scores. This scale demonstrates excellent internal consistency $(\alpha=$

219 0.89; Simons and Gaher, 2005; Peterson et al., 2014). Internal consistencies were adequate to

220 excellent in the current sample (Global Score, $\alpha=0.93, \omega=0.93$; Tolerance, $\alpha=0.84, \omega=0.85$;

221 Appraisal, $\alpha=0.85, \omega=.0 .86$; Absorption $\alpha=0.86, \omega=0.86$; Regulation, $\alpha=0.76, \omega=0.79$ ).

222 Non-suicidal self-injury. Section I of the Inventory of Statements About Self-Injury (ISAS;

223 Klonsky \& Glenn, 2009) was used to assess history and frequency of NSSI (defined to

224 participants as intentionally harming oneself without intention to suicide). Individuals were first

225 provided with a definition of NSSI and then asked "Have you ever engaged in non-suicidal self-

226 injury?". Participant who responded yes to this question were then asked to report a lifetime

227 frequency of twelve common methods of NSSI (e.g. cutting, scratching, burning). The ISAS

228 demonstrates good four week test-retest reliability, $(r=.85$; Klonsky \& Olino, 2008). 


\section{Data analysis}

230 To determine the best fitting model, we examined the model fit of the original higher-order

231

232

233

234

235

236

237

238

239

240

241

242

243

244

245

246

247

248

249

250

251

252

253

254

255

256

257

258

259

260

261

262

263

264

265 factor structure and the lower-order four factor structure of the Distress Tolerance Scale using a sequence of Confirmatory Factor Analyses with a Maximum Likelihood Estimation with robust standard errors and a mean- and variance adjusted test statistic (MLMV). These analyses were conducted among the total sample, the sub-sample of individuals with a history of NSSI, and the sub-sample of individuals without a history of NSSI. A model demonstrated acceptable fit if it met the following criteria: Comparative Fit Index (CFI) and Tucker-Lewis Index (TLI) values between 0.90 (adequate) and 0.95 (good) or higher, and a Standardized Root Mean Square Residual (SRMR) and Root Mean Square Error of Approximation (RMSEA) values close to or below 0.08 (Brown, 2015). Modification indices suggested residual variances be correlated to improve model fit. Given there was a cluster of items with error covariances above .40 on the same subscale, we had theoretical justification for allowing these items to correlate in order to improve model fit (Whittaker, 2012). A chi-square difference test was conducted to statistically compare the two models.

We then tested for measurement invariance across individuals with and without a history of NSSI using the best-fitting model using a multigroup confirmatory factor analysis (MGCFA) with Maximum Likelihood Estimation with robust standard errors and a mean- and variance adjusted test statistic (MLMV). We assessed configural (i.e., equal pattern of factor loadings), metric (i.e., equal factor loadings), scalar (i.e., equal factor loadings and equal intercepts), and residual error invariance (i.e., equal factor loadings, equal intercepts, and equal residual error variance uniqueness). Measurement invariance was supported if the configural model demonstrated acceptable fit and each of the subsequent models showed a non-significant change in chi-square test statistic and a change in Comparative Fit Index (CFI) of $<0.01$ and in Root Mean Square Error of Approximation (RMSEA) of $<0.015$ and Standardized Root Mean Square Residual (SRMR) of $<0.030$ (for metric invariance) or $<0.015$ (for scalar or residual invariance; Chen, 2007) from the previous levels. Partial invariance will be addressed using a sequential backwards approach where items are freed until partial invariance is achieved (Putnick, 2016). All analyses were conducted using MPlus v7.4 (Muthén \& Muthén, 2012)․․

\section{Results}

Results from a Missing Values Analysis indicated data were missing not completely at random, $\chi 2(4012)=4204.185, \mathrm{p}=.02$, however given less than $5 \%$ of data were missing on all variables, Expectation Maximization was used to impute missing data (Tabachnick \& Fidell, 2013). Of the 531 participants, 215 (40.5\%) reported a history of NSSI, with $118(54.9 \%)$ of these individuals reporting engaging in self-injury in the past 12 months. The primary method of NSSI was cutting $(50 \%)$, followed by severe scratching $(12.4 \%)$, and self-battery (11.4\%). Age of onset ranged from 4 to 23 years $(M=13.69, S D=2.99)$. Descriptive statistics are presented in Table 1.

\footnotetext{
${ }^{1}$ Given the majority of participants were female, and at the request of an anonymous reviewer, we also tested measurement invariance across gender. Results indicated that configural, metric, scalar, and residual error invariance across gender were all supported, and thus we conclude males and females do not respond differently to items on this scale (see Supplementary Table 2).
} 


\section{6 (Table 1 approximately here)}

267 Factor structure evaluation. Both the original higher-order model and the lower-order four

268 factor model demonstrated adequate baseline fit in the total sample and among individuals

269 without a history of NSSI, but demonstrated poorer fit among individuals with a history of NSSI

270 (Table 2). Given item 7 "My feelings of distress or being upset are not acceptable", and 11 "I am

271

272

273

274

275

276

277

278

279

280

281

282

283

284

285

286

287

288

289

290

291

292

293

294

295

296

297

298

299

300

301

302

303

304

305

306

307 ashamed of myself when I feel distressed or upset" were both on the appraisal subscale and had error covariance larger than 0.40 , these two items were allowed to correlate. Item 11 and 12 "My feelings of distress or being upset scare me" were also on the appraisal subscale and had error variance larger than 0.40 so were allowed to correlate.

Results from chi-square difference tests indicate allowing these items to correlate significantly improved the baseline fit of the higher-order model in the total sample $\Delta \chi^{2}(2)=75.561, p<.001$, within the sub-sample of individuals with a history of NSSI $\Delta \chi^{2}(2)=24.355, p<.001$, and within the sub-sample of individuals without a history of NSSI $\Delta \chi^{2}(2)=47.708, p<.001$. Despite this improvement, the higher-order model still remained a poor fit among individuals with a history of NSSI (Table 2).

Comparatively, these modifications resulted in the lower-order four factor model demonstrating good fit within the total sample $\Delta \chi^{2}(2)=67.501, \mathrm{p}<.001$, and among individuals without a history of NSSI $\Delta \chi^{2}(2)=43.334, p<.001$, and adequate fit among individuals with a history of NSSI, $\Delta \chi^{2}(2)=21.971, p<.001$. Importantly, chi-square difference tests indicated the lowerorder four factor model was a significantly better fit than the higher-order model in all three groups (Table 2). The factor loadings (full sample) for both the higher-order and lower-order factor models are illustrated in Figure 1 and Figure 2.

\section{(Insert Table 2, Figure 1, and Figure 2 approximately here)}

Measurement invariance of the DTS lower order 4 factor model. Given the correlated lowerorder four factor model was the best fit, this is the model we chose to evaluate for measurement invariance. Configural (M1) and full metric (M2) invariance was supported for the lower-order four factor model, but the $\Delta \chi^{2}$ test statistic indicated full scalar (M3.1) invariance was not supported (Table 3). To address achieve partial invariance, we identified the source of noninvariance by sequentially releasing item intercept constraints until the model was invariant (Putnick et al., 2016). We identified that releasing item 10 intercept constraints had the most influential impact on model fit. Irrespective of the score on the underlying latent factor appraisal, there was a tendency for young adults who self-injured to agree more with item 10 "Being distressed or upset is always a major ordeal for me" $\left(\right.$ Intercept $_{(\mathrm{No} \mathrm{NSSI})}=3.31 \mathrm{vs}$. Intercept $_{(\mathrm{NSSI})}=$ 2.56). Allowing these intercepts to vary between groups, partial scalar (M3.2) and full residual error (M4) invariance was supported.

\section{(Insert Table 3 approximately here)}

There were significant latent mean differences, with individuals with a history of NSSI scoring lower than those with no history on the tolerance subscale $(Z=-7.92, p<.001)$, absorption subscale $(Z=-10.20, p<.001)$, and regulation subscale $(Z=-4.52, p<.001)$. Regardless of whether the differential item functioning of item 10 on the appraisal subscale was considered $(Z$ 
$308=-8.08, p<.001)$, or ignored $(Z=-7.68, p<.001)$, individuals with a history of NSSI scored

309 lower than individuals without a history of NSSI.

\section{Discussion}

311 Many studies use the self-report Distress Tolerance Scale to examine group differences in

312 distress tolerance between individuals with and without a history of self-injury (Anestis et al.,

313 2013; Horgan \& Martin, 2016; Lin et al., 2018; Slabbert et al., 2018a). Relative to individuals

314 with no history of self-injury, individuals with a history of NSSI report less global distress

315 tolerance (Anestis et al., 2013; Lin et al., 2018; Slabbert et al., 2018a), with group differences

316 specifically observed on the appraisal and absorption subscales (Horgan \& Martin, 2016;

317 Slabbert, Hasking, Greene, \& Boyes, in press). However, to ensure confidence in these findings

318 it is important that we confirm the psychometric properties, including measurement invariance,

319 of the Distress Tolerance Scale among individuals with and without a history of self-injury. The

320 aim of the current study was to compare the higher-order and the lower-order four factor

321 structure of the scale among a sample of university students, as well as within the sub-samples of

322 individuals with and without a history of NSSI, to determine the best fitting model, and whether

323 this was invariant across individuals with and without a history of NSSI.

324 Results indicated that the lower-order four factor structure demonstrated superior fit in all

325 analyses. Based on these findings, measurement invariance analyses were conducted on the

326 lower-order four factor model, with results indicating full invariance at the configural and metric

327 level, followed by partial scalar and full residual error invariance. Despite freeing one item

328 intercept at the scalar level, observation of latent mean differences indicate that all subscales can

329 be confidently used to assess NSSI-related group differences in distress tolerance.

330 The findings suggest that using the four subscales of the Distress Tolerance Scale including

331 Tolerance, Appraisal, Absorption, and Regulation, as unique predictors of outcomes such as

332 NSSI, as opposed to a single distress tolerance score, may be statistically superior. It is not

333 uncommon for lower-order factor models to demonstrate better fit than when the lower-order

334 factors are forced to load onto a higher-order factor, with similar results evident in self-report

335 measurement of alexithymia (Meganck et al., 2008). However, it is important to acknowledge

336 that the difference between these two models, although significant, was not large. With some

337 minor modifications, the higher-order factor model still demonstrated adequate fit in the full

338 sample and among individuals with no history of self-injury. This higher-order model may prove

339 useful in research contexts where a global distress tolerance score is valuable, perhaps in studies

340 where researchers are interested in a broad range of constructs and require a more simplistic and

341 direct way of assessing distress tolerance.

342 However, the use of the individual subscales may provide a more comprehensive and holistic

343 understanding of key elements underlying distress tolerance and its relationship with

344 psychopathology and behaviours such as NSSI. For example, in one of the few studies that

345 examined associations between the four distress tolerance subscales and NSSI, Horgan and

346 Martin (2009) established that only the appraisal and absorption subscales differentiated people

347 with and without a history of self-injury. Similarly, Slabbert et al (in press) found that the 
348

349

350

351

352

353

354

355

356

357

358

359

360

361

362

363

364

365

366

367

368

369

370

371

372

373

374

375

376

377

378

379

380

381

382

383

384

385

386

387

388

389

appraisal and absorption scales differentiated between individuals who had recently self-injured and individuals who had never self-injured. Their results also indicated that experiencing greater positive affect might protect against negative appraisals of distress. Based on these findings, how one views their distress and how much attention they allocate towards this distress, appear to be more important in predicting NSSI than an individual's perception of the their tolerance or how they attempt to regulate their distress. Employing this four factor model in future research will allows researchers to delve deeper into the relationship between distress tolerance and self-injury and consequently gain a more accurate and nuanced understanding regarding how different aspects of distress tolerance are related to NSSI.

After establishing that the lower-order four factor model was the superior fitting model, we investigated whether it was invariant among individuals with and without a history of NSSI. Our results were promising, with full configural and metric invariance being supported, and after freeing the item intercept for one item ("Being distressed or upset is always a major deal for me"), partial scalar invariance was achieved. Consequently, full residual error invariance was satisfied however this was contingent on the partial scalar model where the intercept constraints for item 10 were released. When examining this item, it does not appear to differ in terms of its content in comparison to other items, such that it fits well within the general concept of perceived tolerance of distress tolerance. If it appeared to assess something more abstract or obscure in comparison to the other items this may explain differences in interpretation however this does not appear to be the case. Another factor thought to impact interpretation of items is whether they are positively or negatively keyed (Meganck et al., 2008). Previous research has established noninvariance between groups on negatively keyed items (Lindwall et al., 2012), however once again this is not the case with regards to item 10 on the Distress Tolerance Scale as it is positively keyed. Therefore it is not clear why individuals with a history of NSSI may have a different interpretation of this item compared to individuals who have never self-injured. However, the strictest test of invariance was employed in this analysis (chi-square difference test). If we had employed the more liberal criteria used to assess measurement invariance which supports invariance if the difference in CFI between the configural level and other levels is less than 0.01 (Chen, 2007) then full scalar invariance would have been achieved. Regardless, whether this item intercept was freed or not, there were still significant mean differences on the appraisal subscale with individuals with a history of NSSI tending to appraise their distress as more unacceptable than individuals with no history of NSSI. These results instil confidence that we are able to reliably detect real group differences in distress tolerance between individuals with and without a history of NSSI using this four-factor Distress Tolerance Scale.

Whilst the findings of this study provide promising support for the use of the Distress Tolerance Scale to examine NSSI-related group differences in distress tolerance, there are several limitations that warrant consideration. The sample predominantly comprised female university students who self-selected into the study, meaning these findings may not generalise to a community sample. Additionally, while NSSI is prevalent amongst university students, it is unlikely that many would meet the diagnostic criteria for the proposed NSSI disorder (Keikens et al., 2018). Individuals who meet this criteria would likely experience significantly greater difficulties in emotion regulation and consequently may also have more difficulty reflecting on 
390 previous times of heightened distress and reporting on their ability to tolerate distress. Therefore,

391 investigation of measurement invariance of the Distress Tolerance Scale in clinical samples is

392 warranted.

\section{Conclusion}

394 In evaluating the two models of the Distress Tolerance Scale, as well as testing the measurement 395 invariance of the lower-order four factor model, this study has provided support for the use of

396 this scale to reliably assess NSSI-related group differences. The lower-order four factor model

397 appears to be statistically superior and may offer a more comprehensive understanding of the 398 relationship between the specific facets of distress tolerance and behaviours such as NSSI.

399 Additionally, the lower-order four factor model demonstrated invariance up until the scalar level 400 according to the strictest invariance criteria, requiring only one item intercept to be freed to

401 satisfy partial and full residual error invariance. Although further investigation in other samples 402 is required, these results suggest that the Distress Tolerance Scale can be used with confidence 403 that true group differences will be reflected in scores. 
404

405

406

407

408

409

410

411

412

413

414

415

416

417

418

419

420

421

422

423

424

425

426

427

428

429

430

431

432

433

434

435

436

437

438

439

440

441

442

443

444

445

446

447

448

\section{References}

Anestis, M. D., Knorr, A. C., Tull, M. T., Lavender, J. M., \& Gratz, K. L. (2013). The importance of high distress tolerance in the relationship between nonsuicidal self-injury and suicide potential. Suicide and Life Threatening Behaviour, 43(6), 663-675. doi:10.1111/sltb. 12048

Armey, M. F., Crowther, J. H., \& Miller, I. W. (2011). Changes in Ecological Momentary Assessment Reported Affect Associated With Episodes of Nonsuicidal Self-Injury. Behav Ther, 42(4), 579-588. doi:10.1016/j.beth.2011.01.002

Bagby, R. M., Taylor, G. J., Parker, J. D. A., \& Dickens, S. E. (2006). The Development of the Toronto Structured Interview for Alexithymia: Item Selection, Factor Structure, Reliability and Concurrent Validity. Psychother Psychosom, 75(1), 25-39. doi:10.1159/000089224

Boyes, M. E., Wilmot, A., \& Hasking, P. A. (2019). Nonsuicidal Self-Injury-Related Differences in the Experience of Negative and Positive Emotion. Suicide Life Threat Behav. doi: $10.1111 /$ sltb. 12599

Bresin, K. (2014). Five indices of emotion regulation in participants with a history of nonsuicidal self-injury: a daily diary study. Behav Ther, 45(1), 56-66. doi:10.1016/j.beth.2013.09.005

Brown, T. (2015). Confirmatory factor analysis for applied research (2nd ed.). New York: Guildford Press.

Chapman, A. L., Gratz, K. L., \& Brown, M. Z. (2006). Solving the puzzle of deliberate selfharm: the experiential avoidance model. Behav Res Ther, 44(3), 371-394. doi:10.1016/j.brat.2005.03.005

Garnefski, N., \& Kraaij, V. (2007). The Cognitive Emotion Regulation Questionnaire. Psychometric features and prospective relationships with depression and anxiety in adults. European Journal of Psychological Assessment, 23, 141-149.

Gong, T., Ren, Y., Wu, J., Jiang, Y., Hu, W., \& You, J. (2019). The associations among selfcriticism, hopelessness, rumination, and NSSI in adolescents: A moderated mediation model. J Adolesc, 72, 1-9. doi:10.1016/j.adolescence.2019.01.007

Gratz, K., Breetz, A., \& Tull, M. (2010). The moderating role of borderline personality in the relationships between deliberate self-harm and emotion-related factors. Personality and mental health, 4(2), 96-107. doi:10.1002/pmh.102

Gratz, K., \& Roemer, L. (2004). Multidimensional Assessment of Emotion Regulation and Dysregulation: Development, Factor Structure, and Initial Validation of the Difficulties in Emotion Regulation Scale. Journal of Psychopathology and Behavioral Assessment, 26(1), 41-54. doi:10.1023/B:JOBA.0000007455.08539.94

Greene, D., Hasking, P., Boyes, M., \& Preece, D. (2020). Measurement Invariance of Two Measures of Alexithymia in Students Who Do and Who Do Not Engage in Non-suicidal Self-Injury and Risky Drinking. Journal of Psychopathology and Behavioral Assessment. doi:10.1007/s10862-020-09806-7

Gross, J. J., \& John, O. P. (2003). Individual Differences in Two Emotion Regulation Processes: Implications for Affect, Relationships, and Well-Being. Journal of Personality and Social Psychology, 85(2), 348-362. doi:10.1037/0022-3514.85.2.348

Hasking, P., Whitlock, J., Voon, D., \& Rose, A. (2017). A cognitive-emotional model of NSSI: using emotion regulation and cognitive processes to explain why people self-injure. Cogn Emot, 31(8), 1543-1556. doi:10.1080/02699931.2016.1241219 
449

450

451

452

453

454

455

456

457

458

459

460

461

462

463

464

465

466

467

468

469

470

471

472

473

474

475

476

477

478

479

480

481

482

483

484

485

486

487

488

489

490

491

492

493

Horgan, M., \& Martin, G. (2016). Differences between Current and Past Self-Injurers: How and Why Do People Stop? Arch Suicide Res, 20(2), 142-152. doi:10.1080/13811118.2015.1004479

Hovrud, L., Simons, R., Simons, J., \& Korkow, J. (2019). Non-suicidal self-injury and bulimia: the role of emotion dysregulation and body dissatisfaction. Eat Weight Disord, 25(4), 1089-1097. doi:10.1007/s40519-019-00741-5

Jenkins, A. L., \& Schmitz, M. F. (2012). The roles of affect dysregulation and positive affect in non-suicidal self-injury. Arch Suicide Res, 16(3), 212-225. doi:10.1080/13811118.2012.695270

Kiekens, G., Hasking, P., \& Boyes, M. (2019). Measurement Invariance of Three Brief Emotion Regulation Questionnaires in People With and Without a History of Non-Suicidal SelfInjury. European journal of psychological assessment : official organ of the European Association of Psychological Assessment, 35(6), 835-841. doi:10.1027/10155759/a000464

Klonsky, E. D., \& Olino, T. M. (2008). Identifying clinically distinct subgroups of self-injurers among young adults: a latent class analysis. J Consult Clin Psychol, 76(1), 22-27. doi:10.1037/0022-006X.76.1.22

Leyro, T., Bernstein, A., Vujanovic, A., McLeish, A., \& Zvolensky, M. (2010). Distress Tolerance Scale: A Confirmatory Factor Analysis Among Daily Cigarette Smokers. $J$ Psychopathol Behav Assess, 33(1), 47-57. doi:10.1007/s10862-010-9197-2

Leyro, T., Zvolensky, M., \& Bernstein, A. (2010). Distress tolerance and psychopathological symptoms and disorders: a review of the empirical literature among adults. Psychol Bull, 136(4), 576-600. doi:10.1037/a0019712

Lin, M. P., You, J., Wu, Y. W., \& Jiang, Y. (2018). Depression Mediates the Relationship Between Distress Tolerance and Nonsuicidal Self-Injury Among Adolescents: One-Year Follow-Up. Suicide Life Threat Behav, 48(5), 589-600. doi:10.1111/sltb.12382

Lindwall, M., Barkoukis, V., Grano, C., Lucidi, F., Raudsepp, L., Liukkonen, J., \& ThøgersenNtoumani, C. (2012). Method Effects: The Problem With Negatively Versus Positively Keyed Items. J Pers Assess, 94(2), 196-204. doi:10.1080/00223891.2011.645936

10.1037/0003-066x.54.3.165

Meganck, R., Vanheule, S., \& Desmet, M. (2008). Factorial Validity and Measurement Invariance of the 20-Item Toronto Alexithymia Scale in Clinical and Nonclinical Samples. Assessment, 15(1), 36-47. doi:10.1177/1073191107306140

Najmi, S., Wegner, D. M., \& Nock, M. K. (2007). Thought suppression and self-injurious thoughts and behaviors. Behav Res Ther, 45(8), 1957-1965. doi:10.1016/j.brat.2006.09.014

Peterson, C. M., Davis-Becker, K., \& Fischer, S. (2014). Interactive role of depression, distress tolerance and negative urgency on non-suicidal self-injury. Personal Ment Health, 8(2), 151-160. doi:10.1002/pmh.1256

Putnick, D., \& Bornstein, M. (2016). Measurement invariance conventions and reporting: The state of the art and future directions for psychological research. Dev Rev, 41, 71-90. doi:10.1016/j.dr.2016.06.004

Sandín, B., Simons, J. S., Valiente, R. M., Simons, R. M., \& Chorot, P. (2017). Psychometric properties of the spanish version of The Distress Tolerance Scale and its relationship with personality and psychopathological symptoms. Psicothema, 29(3), 421. 
494

495

496

497

498

499

500

501

502

503

504

505

506

507

508

509

510

511

512

513

514

515

516

517

518

519

520

521

522

523

524

525

526

527

528

529

530

Sass, D. (2016). Testing Measurement Invariance and Comparing Latent Factor Means Within a Confirmatory Factor Analysis Framework. Journal of psychoeducational assessment, 29(4), 347-363. doi:10.1177/0734282911406661

Selby, E. A., Anestis, M. D., \& Joiner, T. E. (2008). Understanding the relationship between emotional and behavioral dysregulation: emotional cascades. Behav Res Ther, 46(5), 593611. doi:10.1016/j.brat.2008.02.002

Simons, S. J., \& Gaher, M. R. (2005). The distress tolerance scale: development and validation of a self-report measure. Motiv. Emot., 29(2), 83-102.

Slabbert, A., Hasking, P., \& Boyes, M. (2018a). Riding the emotional roller coaster: The role of distress tolerance in non-suicidal self-injury. Psychiatry Res, 269, 309-315. doi:10.1016/j.psychres.2018.08.061

Slabbert, A., Hasking, P., \& Boyes, M. (2018b). Riding the emotional roller coaster: The role of distress tolerance in nonsuicidal self-injury. Psychiarty Research, 269, 309-315. doi:https://doi.org/10.1016/i.psychres.2018.08.061

Slabbert, A., Hasking, P., Greene, D., \& Boyes, M. (in press). The role of distress tolerance in the relationship between affect and NSSI. Archives of Suicide Research.

Slabbert, A., Hasking, P., Notebaert, L., \& Boyes, M. (2020). The Role of Distress Tolerance in the Relationship Between Affect and NSSI. Archives of Suicide Research, 1-15. doi:10.1080/13811118.2020.1833797

Swannell, S. V., Martin, G. E., Page, A., Hasking, P., \& St John, N. J. (2014). Prevalence of nonsuicidal self-injury in nonclinical samples: systematic review, meta-analysis and meta-regression. Suicide Life Threat Behav, 44(3), 273-303. doi:10.1111/sltb.12070

Tabachnick, B. G., \& Fidell, L. S. (2013). Using Multivariate Statistics. Pearson, Boston.

Taylor, P. J., Jomar, K., Dhingra, K., Forrester, R., Shahmalak, U., \& Dickson, J. M. (2018). A meta-analysis of the prevalence of different functions of non-suicidal self-injury. $J$ Affect Disord, 227, 759-769. doi:10.1016/j.jad.2017.11.073

Victor, S. E., \& Klonsky, E. D. (2014). Daily emotion in non-suicidal self-injury. J Clin Psychol, 70(4), 364-375. doi:10.1002/jclp.22037

Werner-Seidler, A., Banks, R., Dunn, B. D., \& Moulds, M. L. (2013). An investigation of the relationship between positive affect regulation and depression. Behaviour Research and Therapy, 51(1), 46-56. doi:10.1016/j.brat.2012.11.001

Whitlock, J., Muehlenkamp, J., Eckenrode, J., Purington, A., Baral Abrams, G., Barreira, P., \& Kress, V. (2013). Nonsuicidal self-injury as a gateway to suicide in young adults. $J$ Adolesc Health, 52(4), 486-492. doi:10.1016/j.jadohealth.2012.09.010

Whittaker, T. (2012). Using the Modification Index and Standardized Expected Parameter Change for Model Modification. The Journal of experimental education, 80(1), 26-44. doi:10.1080/00220973.2010.531299 
Table $\mathbf{1}$ (on next page)

Descriptive statistics disaggregated by history of NSSI 
Table 1

Descriptive statistics disaggregated by history of NSSI

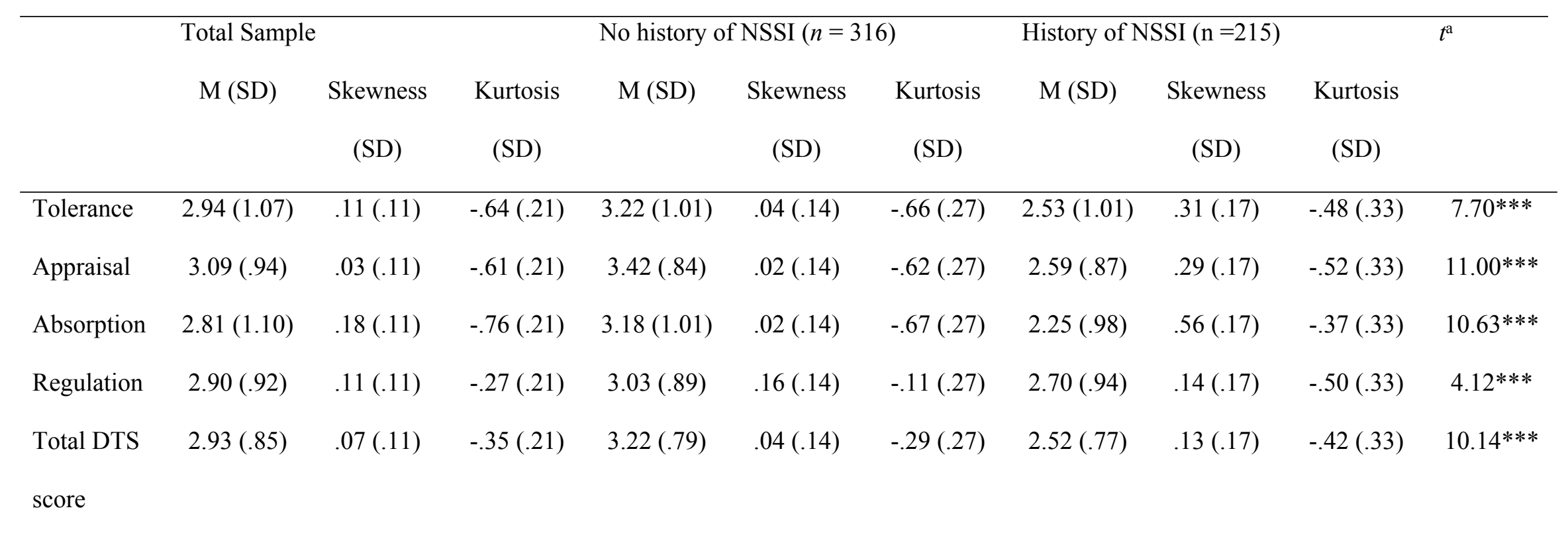

${ }^{a} \mathrm{t}$ values are in reference to the mean comparison between individuals with and without a history of NSSI.

${ }^{*} p<.05 .{ }^{* *} p<.01 .{ }^{* * *} p<.001$.

1

2

3

4 


\section{Table 2(on next page)}

Comparison of DTS models among the total sample, individuals with a history of NSSI, and individuals without a history of NSSI 
Table 2

Comparison of DTS models among the total sample, individuals with a history of NSSI, and individuals without a history of NSSI

\begin{tabular}{|c|c|c|c|c|c|c|c|c|}
\hline & $\chi^{2}$ & $d f$ & $\Delta \chi^{2}(\Delta \mathrm{df})$ & $p \Delta \chi^{2}$ & CFI & TLI & RMSEA & SRMR \\
\hline \multicolumn{9}{|l|}{ Total sample $(n=531)$} \\
\hline \multicolumn{9}{|l|}{ Baseline fit } \\
\hline Higher-order factor model & 356.534 & 86 & - & - & 0.915 & 0.896 & 0.077 & 0.059 \\
\hline Lower-order factor model & 312.991 & 82 & $43.543(2)$ & $<0.001$ & 0.928 & 0.910 & 0.072 & 0.051 \\
\hline \multicolumn{9}{|l|}{$\begin{array}{l}\text { Baseline fit with appraisal item } \\
\text { correlations }\end{array}$} \\
\hline Higher-order factor model & 280.973 & 84 & - & - & 0.938 & 0.923 & 0.066 & 0.055 \\
\hline Lower-order factor model & 245.490 & 82 & $35.483(2)$ & $<0.001$ & 0.949 & 0.934 & 0.061 & .047 \\
\hline \multicolumn{9}{|l|}{ NSSI history $(n=215)$} \\
\hline \multicolumn{9}{|l|}{ Baseline fit } \\
\hline Higher-order factor model & 210.779 & 86 & - & & 0.872 & 0.844 & 0.082 & 0.087 \\
\hline Lower-order factor model & 181.950 & 84 & $28.829(2)$ & $<0.001$ & 0.899 & 0.874 & 0.074 & 0.071 \\
\hline \multicolumn{9}{|l|}{$\begin{array}{l}\text { Baseline fit with appraisal item } \\
\text { correlations }\end{array}$} \\
\hline Higher-order factor model & 186.424 & 84 & - & - & 0.895 & 0.869 & 0.075 & 0.084 \\
\hline Lower-order factor model & 159.979 & 82 & $26.45(2)$ & $<0.001$ & 0.920 & 0.897 & 0.067 & 0.067 \\
\hline \multicolumn{9}{|l|}{ No NSSI history $(n=316)$} \\
\hline \multicolumn{9}{|l|}{ Baseline fit } \\
\hline Higher-order factor model & 200.891 & 86 & - & & 0.923 & 0.906 & 0.065 & 0.053 \\
\hline Lower-order factor model & 188.532 & 84 & $12.359(2)$ & 0.002 & 0.930 & 0.913 & 0.063 & 0.051 \\
\hline \multicolumn{9}{|l|}{$\begin{array}{l}\text { Baseline fit with appraisal item } \\
\text { correlations }\end{array}$} \\
\hline Higher-order factor model & 153.183 & 84 & - & - & 0.954 & 0.942 & 0.051 & 0.049 \\
\hline Lower-order factor model & 145.198 & 82 & $7.99(2)$ & 0.018 & 0.958 & 0.946 & 0.049 & 0.045 \\
\hline
\end{tabular}




\section{Table 3 (on next page)}

Measurement invariance of the lower-order four factor Distress Tolerance Scale 
Table 3

Measurement invariance of the lower-order four factor Distress Tolerance Scale

\begin{tabular}{|c|c|c|c|c|c|c|c|c|c|c|c|c|c|}
\hline & $\chi^{2}$ & $d f$ & $\Delta \chi^{2}(\Delta \mathrm{df})$ & $p \Delta \chi^{2}$ & $\mathrm{NCI}$ & CFI & RMSEA & SRMR & $\begin{array}{c}\text { Model } \\
\text { Comparison }\end{array}$ & $\triangle \mathrm{NCI}$ & $\Delta \mathrm{CFI}$ & $\triangle \mathrm{RMSEA}$ & $\Delta \mathrm{SRMR}$ \\
\hline $\begin{array}{l}\text { Model 1: } \\
\text { Configural } \\
\text { invariance }\end{array}$ & 304.965 & 164 & - & - & 0.8755 & 0.943 & 0.057 & 0.055 & - & - & - & - & - \\
\hline $\begin{array}{l}\text { Model 2: } \\
\text { Full metric } \\
\text { invariance }\end{array}$ & 315.623 & 175 & $10.66(11)$ & 0.472 & 0.8758 & 0.943 & 0.055 & 0.058 & M1-M2 & 0.0003 & $<0.001$ & 0.002 & 0.003 \\
\hline $\begin{array}{l}\text { Model 3.1: } \\
\text { Full scalar } \\
\text { invariance }\end{array}$ & 338.147 & 186 & $22.52(11)$ & 0.021 & 0.8663 & 0.939 & 0.056 & 0.063 & M2-M3.1 & 0.0100 & 0.004 & 0.001 & 0.005 \\
\hline $\begin{array}{l}\text { Model 3.2: } \\
\text { Partial scalar } \\
\text { invariance }\end{array}$ & 328.614 & 185 & $12.99(10)$ & 0.224 & 0.8733 & 0.942 & 0.054 & 0.060 & M2-M3.2 & 0.0035 & 0.001 & 0.001 & 0.002 \\
\hline $\begin{array}{l}\text { Model 4: } \\
\text { Full residual } \\
\text { error } \\
\text { invariance }\end{array}$ & 348.770 & 200 & $20.16(15)$ & 0.166 & 0.8691 & 0.940 & 0.053 & 0.062 & M3.2-M4 & 0.0042 & 0.002 & 0.001 & 0.002 \\
\hline 1 & & & & & & & & & & & & & \\
\hline 2 & & & & & & & & & & & & & \\
\hline 3 & & & & & & & & & & & & & \\
\hline
\end{tabular}


Figure 1

\section{Distress Tolerance Scale Higher-Order Factor Model (Total Sample)}

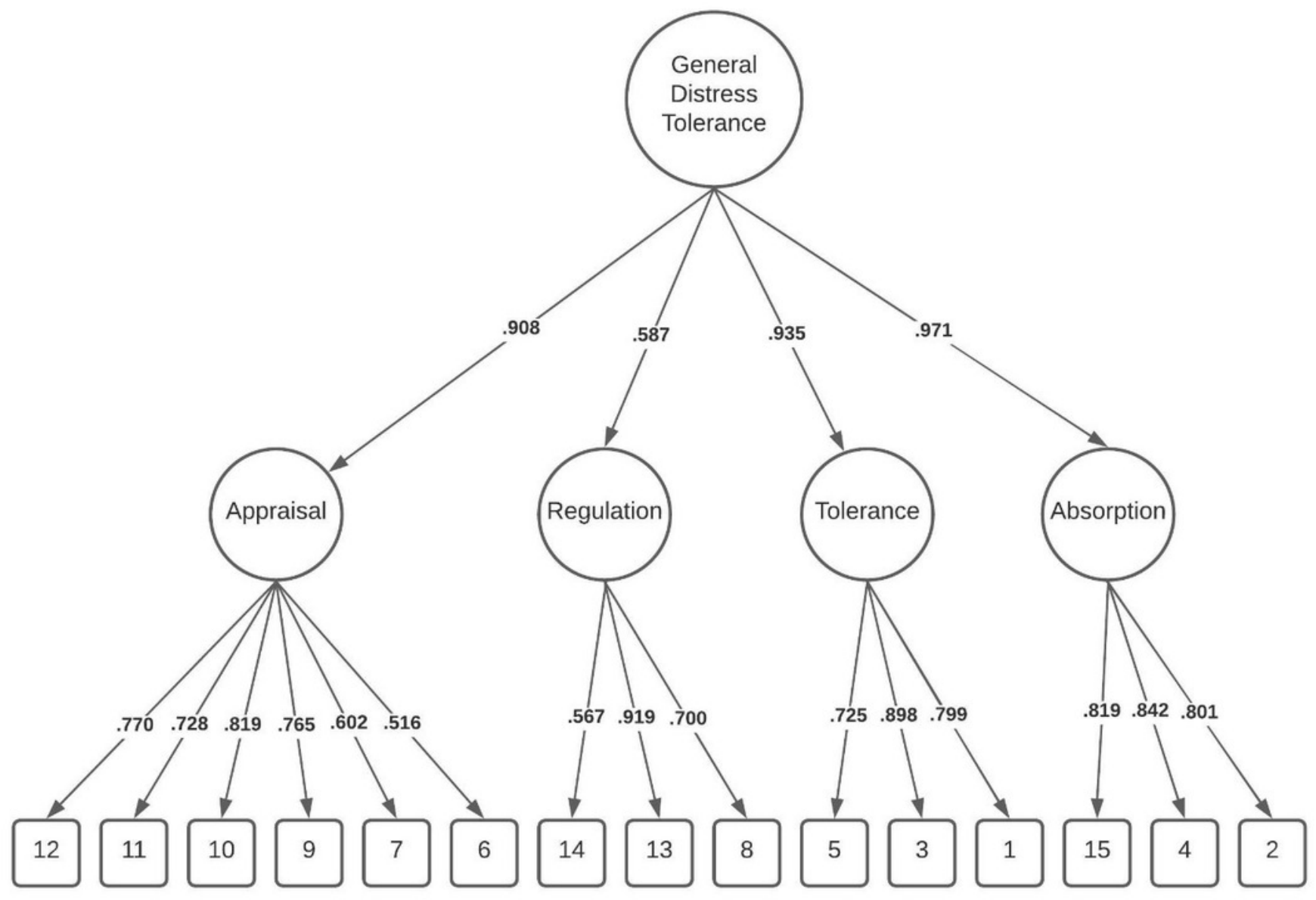


Figure 2

Distress Tolerance Scale Lower-Order Four Factor Model (Total Sample)

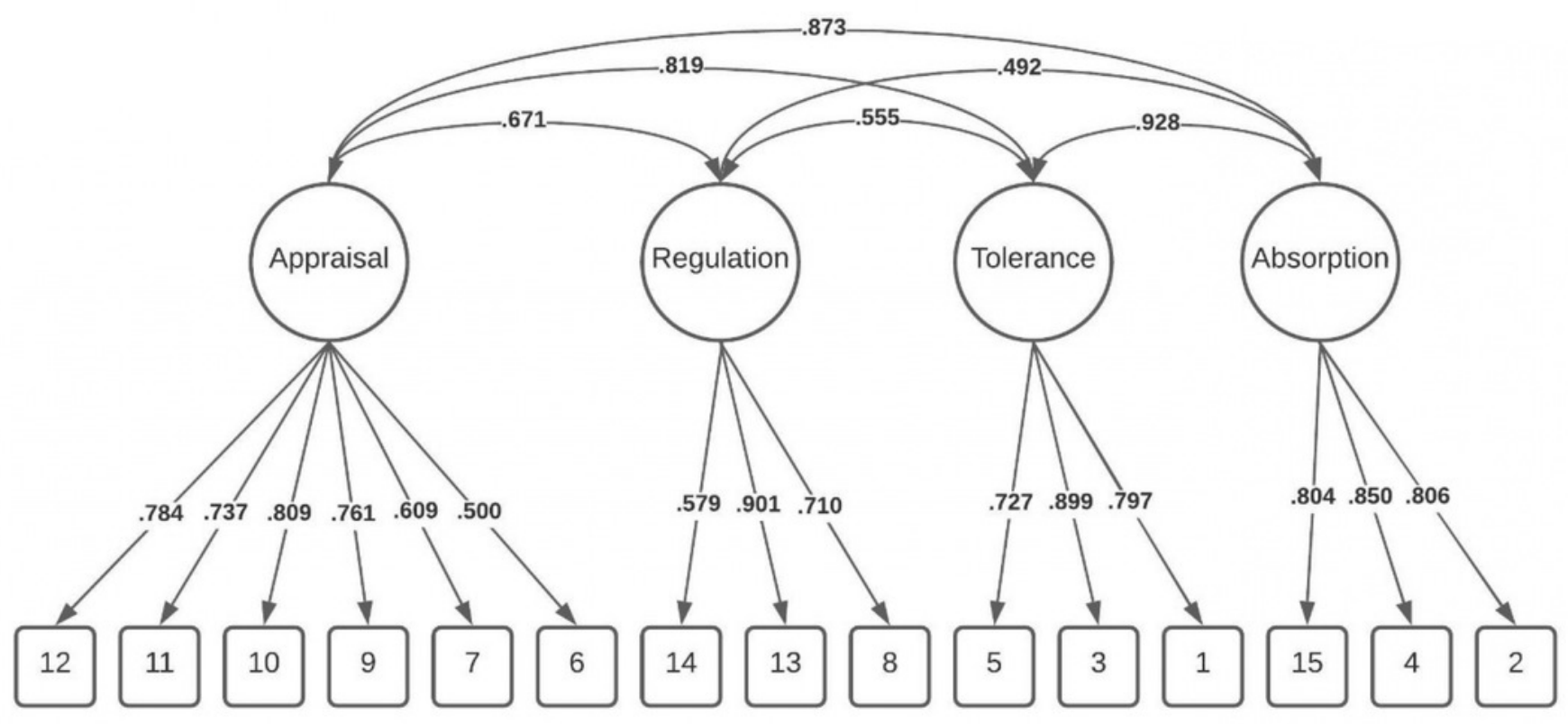

